\title{
GLAD!
}

Revue sur le langage, le genre, les sexualités

$06 \mid 2019$

Varia

\section{Déconstruction des injonctions de genre}

Écrivaines hispano-américaines

Deconstructing Gender Injunctions: Latin-American Female Writers

\section{María Jesús Fariña Busto}

Traducteur : Marion Marchetti

\section{(2) OpenEdition}

Journals

Édition électronique

URL : http://journals.openedition.org/glad/1467

DOI : $10.4000 /$ glad. 1467

ISSN : 2551-0819

Éditeur

Association GSL

Référence électronique

María Jesús Fariña Busto, « Déconstruction des injonctions de genre », GLAD! [En ligne], 06 | 2019, mis en ligne le 01 juillet 2019, consulté le 17 décembre 2020. URL : http://journals.openedition.org/glad/ 1467 ; DOI : https://doi.org/10.4000/glad.1467

Ce document a été généré automatiquement le 17 décembre 2020.

\section{(c) (i) (9)}

La revue GLAD! est mise à disposition selon les termes de la Licence Creative Commons Attribution Pas d'Utilisation Commerciale - Pas de Modification 4.0 International. 


\title{
Déconstruction des injonctions de
}

\section{genre}

\author{
Écrivaines hispano-américaines \\ Deconstructing Gender Injunctions: Latin-American Female Writers
}

\author{
María Jesús Fariña Busto
}

Traduction : Marion Marchetti

\section{RÉFÉRENCE}

FARIÑA BUSTO, María Jesús. 2013. « Desactuando el mandato de género: escritoras hispanoamericanas » Sociocriticism [En ligne], 28. URL : http://revistaseug.ugr.es/ index.php/sociocriticism/article/view/2397

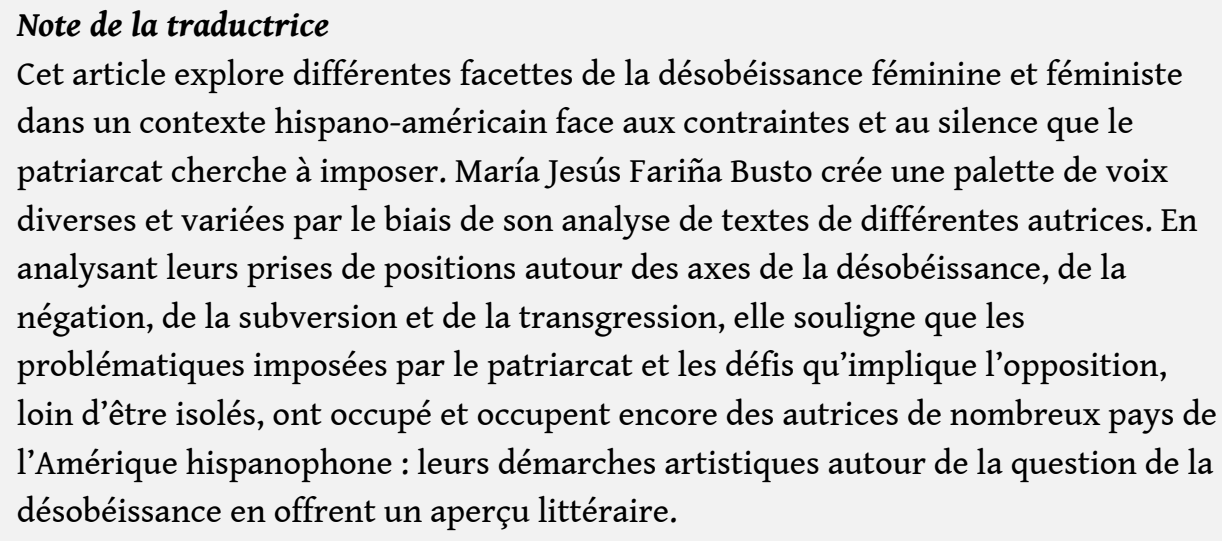

Daquelas que cantan as pombas e as frores, todos din que teñen alma de muller. Pois eu que n'as canto, Virxe da Paloma, ¡ai! ¿de qué a terei? De celles qui chantent les colombes et les fleurs, 
on dit qu'elles ont âmes féminines.

hélas! Vierge de la Colombe, je ne les chante

point :

quel âme aurais-je?

Rosalía de Castro (De Castro $2002: 63$ )

El feminismo no ha muerto, porque sólo muere lo

finalizado, lo acabado, lo inerme.

Le féminisme n'est pas mort, car seul peut mourir ce qui est finalisé, bouclé, inerte. ${ }^{1}$

Iris M. Zavala

1 Désobéir est un verbe que les femmes ont toujours conjugué. La création artistique en est une forme : transgresser la loi du père, déjouer les normes contraignantes et anticulturelles; refuser de reconnaitre les paramètres que la culture impose comme allant de soi, ou exige que l'on sache interpréter; en définitive, déconstruire les injonctions, vivre dans la résistance. L'histoire le prouve malgré les silences et les dissimulations. À toutes les époques, les femmes se sont exprimées dans les milieux érudits et artistiques, bien que leurs exploits aient été occultés ou discrédités, et bien que cette prise de parole les ait mises en danger de mort. Contre la mort, la création; et contre la violence, toujours plus de désobéissance.

Dans ses deux courants pratiques et théoriques, le féminisme a favorisé la coalition de plusieurs moyens d'expression et a influencé diversement ses modes et manifestations, en infiltrant même les attitudes et comportements de personnes qui manifestent leur détachement de ce mouvement. En 1996, Eliana Ortega² écrivait déjà :

Le féminisme est aujourd'hui bel et bien présent, même si les langages du pouvoir et les personnes qui y sont soumises cherchent à l'occulter; il est présent en pratique et en théorie, sous toutes ses formes, ses contradictions, ses doutes, ses interrogations et ses affirmations, dans son immense diversité. (Ortega $1996: 31)^{3}$.

Quinze ans plus tard, il est devenu indispensable de souligner cette présence et sa nécessité, car les discours du pouvoir absorbent tout ce qui leur barre le chemin, qu'ils désirent occulter ou tout simplement supprimer. Sous prétexte que tous les droits seraient déjà obtenus, et avec l'appui de nouveaux discours qui cherchent à affaiblir des revendications qui n'ont pas encore obtenu gain de cause, une représentation biaisée de la réalité se met en place, hystérisant toute action ou expression dissidente ou contestataire. L'histoire prouve aussi que les réclamations des femmes les plus résistantes sont aussi fermes que les signes de répression sont nombreux. Christine Bard le montre dans le contexte historique français en rappelant que le parcours des revendications des femmes au cours du vingtième siècle a été accompagné par les rhétoriques réactionnaires qu'elles ont déclenchées, en entendant le terme réactionnaire au sens strict, de réaction, mais également en tant que rhétoriques ultraconservatrices des privilèges patriarcaux (Bard 2000) ${ }^{4}$. Amélia Valcárcel le formule de la manière suivante : «A chaque revendication d'égalité, l'opposition a riposté avec une naturalisation du sexe, adaptée dans son langage aux conceptualisations de son époque » $(1994: 10)$.

En 1919, Alfonsina Storni soulignait déjà la représentation négative du féminisme en affirmant: «Le mot féministe, "tellement moche" encore aujourd'hui, chatouille toujours les âmes humaines " (Storni rééd. $2002: 839$ ). Montserrat Roig l'a répété en 1981 : «Encore à notre époque, le mot féminisme fait peur. Être "féministe" signifie 
encore, pour certaines femmes, se distancer des hommes, être une femme différente, agressive, menaçant la paix et la vie en communauté » (Bosch et Ferrer $2003: 146$ ). Enfin, en 2006, Ana María Rodas répondait, lors d'une interview : «Je ne fais pas partie des femmes qui pensent qu'il faut exterminer la moitié de la population du monde. Mais oui, je suis féministe, parce qu'une immense partie des femmes de ce monde va très mal » (Montenegro 2006).

En utilisant des mécanismes de discrédit envers ses activistes et ses théoriciennes, le système patriarcal a toujours cherché à dévaloriser et à pervertir les paris et les demandes du mouvement féministe. Il prétendait ainsi se défendre face aux revendications de droits pour tous les êtres humains, les formes d'action et de relation non asphyxiées par les normes de genre, dont les résultats les plus évidents sont la frustration individuelle, le déséquilibre et la violence sociale. Cette perversion s'est également produite dans des contextes où la force et la vie des femmes ont été mises à contribution dans des projets révolutionnaires; l'objectif atteint, leurs droits ont été abandonnés ${ }^{5}$. Le même schéma se met en place du côté de la critique littéraire face aux projets à vocation transgressive. La colombienne Albalucía Ángel utilise avec humour certains des termes qui lui ont été adressés :

[...] l'écriture de mon roman Estaba la pájara pinta sentada en el verde limón (la garce maquillée était assise sur le citron vert) m'aura valu le même surnom. Et ça me plait. Sans compter les sobriquets de tête en l'air (folle furieuse), pornographe, obscène, polémique, controversée, loufoque, extravagante et bien d'autres, par lesquels j'ai été renommée, rebaptisée et reconnue à outrance. (Ángel 1985 : 456)

Ironiquement, ce système qui s'institue en accusateur a lui-même insidieusement cherché à tuer, bien qu'au niveau symbolique seulement (c'est-à-dire oblitérer et réduire au silence), au moins la moitié de la population mondiale. Ce système est une forme de violence symbolique, peut-être la plus puissante, car elle sert de fondement à la manifestation de violences réelles.

7 Dans leur hiérarchisation, les configurations culturelles du masculin et du féminin portent les traces de cette violence symbolique. Les paires sont infinies : bon/mauvais, supérieur/inférieur, indépendant/dépendant, autorité/subordination, pour n'en citer que quelques-unes. Les sujets intériorisent ces configurations en naturalisant ce qui n'est qu'une construction intéressée, donc réfutable et déconstructible. Dans les pages qui suivent, je rassemble quelques exemples littéraires d'autrices hispanoaméricaines qui se positionnent contre les rôles genrés. Il s'agit parfois de littérature suggestive, mais aussi et surtout de textes jouant sur des formules crues et ouvertement subversives. Quant à leur chronologie, la plupart de ces œuvres ont été publiées pendant les quarante dernières années.

\section{Désobéissance}

8 La différence, non pas considérée comme un fait, mais construite comme inégalité, comme dépendance et comme subalternité, a été une injonction culturelle imposée aux sujets féminins. Être la propriété, en premier lieu du père, puis du mari : 


\begin{tabular}{|l|l|}
\hline & ¿Pero quién soy yo? tu esposa, \\
claro. Y ese título basta para & Mais qui suis-je ? Ta femme, \\
distinguirme de los recuerdos del & bien sûr. Et ce titre suffit pour \\
me distinguer des souvenirs du & passé, des projets d'avenir. Je \\
porvenir. Llevo una marca de & porte cette marque de ta \\
propiedad y no obstante me miras & propriété, et pourtant tu me \\
con desconfianza. No estoy & considères avec méfiance. Je \\
tejiendo una red para prenderte. & ne suis pas en train de tisser \\
No soy una mantis religiosa. Te & une toile pour te piéger. Je ne \\
agradezco que creas en semejante & suis pas une mante religieuse. \\
hipótesis. Pero es falsa. & Je te remercie de croire \\
(Castellanos 1971:14) & pareille hypothèse. Mais elle \\
& est fausse. \\
\hline
\end{tabular}

9 C'est la voix de la protagoniste de «Lección de cocina » (Cours de cuisine), de Rosario Castellanos, « abnegada mujercita mexicana que nació como la paloma para el nido » (« petite mexicaine dévouée, utérus sur pattes ») mais qui s'obstine à ne pas savoir lire ce que la culture lui commande de savoir lire en tant que femme : « Me supone [el manual de cocina] una intuición que, según mi sexo, debo poseer pero que no poseo, un sentido sin el que nací que me permitiría advertir el momento preciso en que la carne está a punto» (ibidem: 13) («[Le livre de cuisine] présuppose que mon sexe m'octroie une intuition que je n'ai pas, une sensibilité qui ne m'a pas accompagnée à la naissance, mais qui me permettrait de remarquer à quel moment précis la viande sera cuite à point »).

10 La protagoniste manque de savoir normatif, ou peut-être y résiste-t-elle. Elle possède néanmoins un autre savoir qui se révèle à travers ses commentaires, ses références et son usage de la langue. Une fois sa lune de miel achevée, elle fait face au moment de vérité, celui du tout premier plat à cuisiner en tant qu'épouse. Tout se retourne contre elle : le livre de cuisine qu'elle ne comprend pas, la viande qui finira par bruler dans la casserole, sa propre réflexion qui l'oblige à se confronter à son présent et à son futur. Le récit est rythmé par le flux de sa pensée. Le va-et-vient entre ses réflexions et la réalité (le plat qu'elle prépare dans sa cuisine) illustrent sa position en tant que femme au sein du système patriarcal ${ }^{6}$. L'espace privé domestique de la cuisine acquiert, à partir de cette stratégie, une nouvelle valeur : il apparait comme un lieu de production éthique, esthétique et métaphysique. Sa réflexion fait défiler des évènements de sa jeunesse (ses études, ses projets et ses amitiés), des épisodes de la lune de miel (ses expériences sexuelles, pas vraiment enthousiasmantes), ainsi que des évocations et des associations littéraires et historiques. Tout ceci sous le regard d'une personne qui fait des liens entre ces expériences, de vie et d'apprentissage, et le moment qu'elle passe à la cuisine, qu'elle se représente comme emblématique de ce qui lui est arrivé et de ce qui l'attend.

11 Alors qu'elle retourne encore et encore son morceau de viande, la protagoniste réalise, dans une permanente ironie, que le mariage déclenche un ensemble de pratiques de dépendance et de domination; à commencer par le changement de nom («perdí mi antiguo nombre y aún no me acostumbro al nuevo », 11 - «j'ai perdu mon ancien nom et je ne me suis pas encore habituée au nouveau »), et se prolongeant par la perte de l'autonomie et de la liberté économique. Ce nouveau statut d'épouse s'assimile avant tout à l'esclavage, comme le démontre le terme qui conclut le paragraphe suivant : 


\begin{tabular}{|c|c|}
\hline $\begin{array}{l}\text { Se me atribuyen las } \\
\text { responsabilidades y las tareas de } \\
\text { una criada para todo. He de } \\
\text { mantener la casa impecable, la } \\
\text { ropa lista, el ritmo de la } \\
\text { alimentación infalible. Pero no se } \\
\text { me paga ningún sueldo, no se me } \\
\text { concede un día libre a la semana, } \\
\text { no puedo cambiar de amo. (15) }\end{array}$ & $\begin{array}{l}\text { On m'attribue les tâches et les } \\
\text { responsabilités d'une } \\
\text { domestique pour tout. Je dois } \\
\text { veiller à ce que la maison soit } \\
\text { impeccable, les vêtements } \\
\text { propres et les repas ponctuels. } \\
\text { Mais on ne me paie aucun } \\
\text { salaire, on ne me donne aucun } \\
\text { jour de congé hebdomadaire et } \\
\text { je ne peux pas changer de } \\
\text { maitre. }\end{array}$ \\
\hline
\end{tabular}

Consciente de sa situation, elle évalue les différentes issues de secours imaginables: imposer elle-même les règles du jeu ou suivre «la sinuosa vía que recorrieron mis antepasadas, las humildes, las que no abrían los labios sino para asentir» (21) (« la voie sinueuse qu'ont empruntée mes ancêtres, les humbles, celles qui n'ouvraient jamais la bouche sauf pour acquiescer »). Cette fin ouverte a fait couler beaucoup d'encre chez les critiques littéraires. L'idée de Rosario Castellanos aura su intriguer les esprits. Au fond, l'importance ne réside pas dans le choix final de la protagoniste, mais plutôt dans le fait que cette décision sera prise en connaissance de cause, c'est-à-dire en toute conscience des risques qu'elle engendre ; ce qui n'est pas synonyme de liberté absolue, car la trame culturelle est tissée d'injonctions subtiles et achevées. La fin ouverte projette cette décision en dehors du texte, incitant les lecteurs et lectrices à y participer, ce qui laisse place aux hypothèses concernant les possibilités verbalisées par la protagoniste et les conséquences que chacune d'entre elles aura.

Sortir de la dépendance, de l'appartenance, de la généalogie patrilinéaire, construire une autre échelle, devenir insoumise, chacun de ces mouvements a toujours entrainé une certaine pénalité: la non-reconnaissance, la critique féroce, la frustration, l'isolement, la folie ${ }^{8}$, parfois même une renommée d'exceptionnalité, ce qui, une fois en rigueur, n'a été qu'une autre forme de pénalisation, le sujet féminin étant considéré comme une curiosité face à la majorité qui ne la compte pas parmi ses membres. Cependant, les exemples ne manquent pas, certains canonisés à travers ce que l'on pourrait qualifier de travestisme symbolique, imposé depuis l'extérieur, comme l'excroissance d'une pensée ayant refusé de reconnaitre le talent intellectuel et créatif des femmes. Il s'agissait, dans ce cas-là, d'une femme masculine, sur laquelle la nature se serait trompée. L'espagnole Emilia Pardo Bazán et la cubaine Gertrudis Gómez de Avallenada, on le sait, ont été jugées comme telles. En ce qui concerne la première, un de ses éditeurs, Sainz de Robles, a affirmé que « si en écrivant des romans, Emilia Pardo Bazán n'était 'rien de moins qu'un homme [...]', alors comme chercheuse et critique, elle n'est 'rien de moins qu'une femme'. Mais rien de plus » (Luna 1996: 16). Quant à la seconde, son compatriote José Martí l'a décrite dans les termes suivants : «Il n’y a pas de femme dans le corps de Gómez de Avallenada : tout annonçait un courage puissant et viril en elle $»^{9}$.

14 Il faudrait considérer, en parallèle à cette idée de travestisme symbolique, l'idée envisagée par l'écrivaine galicienne Rosalía de Castro dans les quatre vers que j'ai cités au début de l'article. Elle représente elle-même un cas littéraire singulier: celui d'une autrice qui est devenue une référence canonique de la littérature d'un pays. Mais la façon dont ses contemporains se sont intéressés à son œuvre est aussi singulière, car, jusqu'à la deuxième partie du vingtième siècle, le réflexe le plus habituel était de 
contourner son combat féministe, exposé de manière révélatrice dans la «Carta a Eduarda » ("Lettre à Eduarda ») et dans de nombreux autres textes, dont le poème cité. Sous une apparence simple, ce dernier soulève une question complexe : qui est cette personne qui n'obéit pas, qui ne se plie pas aux conventions que l'institution littéraire définit pour une écrivaine? Quelle âme est attachée à un tel corps ?

\section{Négation}

15 «Las mujeres ya no quieren callar» («Les femmes ne veulent plus se taire ») : c'est ainsi que la péruvienne Doris Monomisato intitule l'un de ses poèmes publié en 2003 (Luna $2004: 330$ ). Bien que le parcours généalogique tracé par le texte, comme l'indique le sous-titre («Prosaica y coloquial travesía por la poética literatura de las mujeres » "Voyage prosaïque familier à travers la poétique littéraire des femmes »), suggère qu'elles ne l'avaient jamais fait, cette affirmation, au sujet de laquelle j'ai déjà insisté, requiert encore d'être accentuée : les écrivaines et les femmes artistes ne sont jamais restées à l'écart des mouvements et des débats de leurs époques. Dans de nombreux cas, elles en ont elles-mêmes été les promotrices; voire dans d'autres, en plus d'être promotrices, également des victimes. Leurs paroles sagaces et engagées fonctionnaient comme signal d'alarme auprès d'un système qui n'était pas disposé à renoncer à ses postulats.

Le poème de Moromisato voit défiler Sœur Juana (la première, évidemment, bien qu'elle ne le soit pas dans l'histoire), les péruviennes Clorinda Matto et Mercedes Cabello, l'uruguayenne Delmira Agustini, la chilienne María Luisa Bombal, l'argentine Alfonsina Storni, la mexicaine Rosario Castellanos, la brésilienne Clarice Lispector ainsi que les péruviennes Blanca Varela et Carmen Luz Bejarano (auxquelles le texte est dédié). Les nommer est un moyen de les délivrer, de reconnaitre leur autorité et leur influence. De plus, leurs différentes chronologies, origines géographiques et sociales dressent une carte de volonté et de complicité, qui se répandent au-delà des frontières et des époques, bien que leurs compromis individuels s'inscrivent dans des circonstances et des dilemmes propres à leurs périodes historiques respectives.

À travers leurs œuvres, toutes ont canalisé des préoccupations, des revendications et des propositions de rébellion, sans négliger de travailler sur ce qui constitue leur matière principale: les différents langages de ceux qui s'en servent. Ce sont des créatrices conscientes d'elles-mêmes et des limites imposées par la culture en raison de leur sexe. Leur force créatrice les élève dans leur capacité d'agir et de transformer, comme le prouvent leurs œuvres qui en sont le résultat.

Néanmoins, dans ce contexte, s'affirmer requiert de commencer par une négation, celle du stéréotype, de la norme. Renoncer à la soumission constitue le premier pas vers l'établissement de nouveaux espaces d'action. 


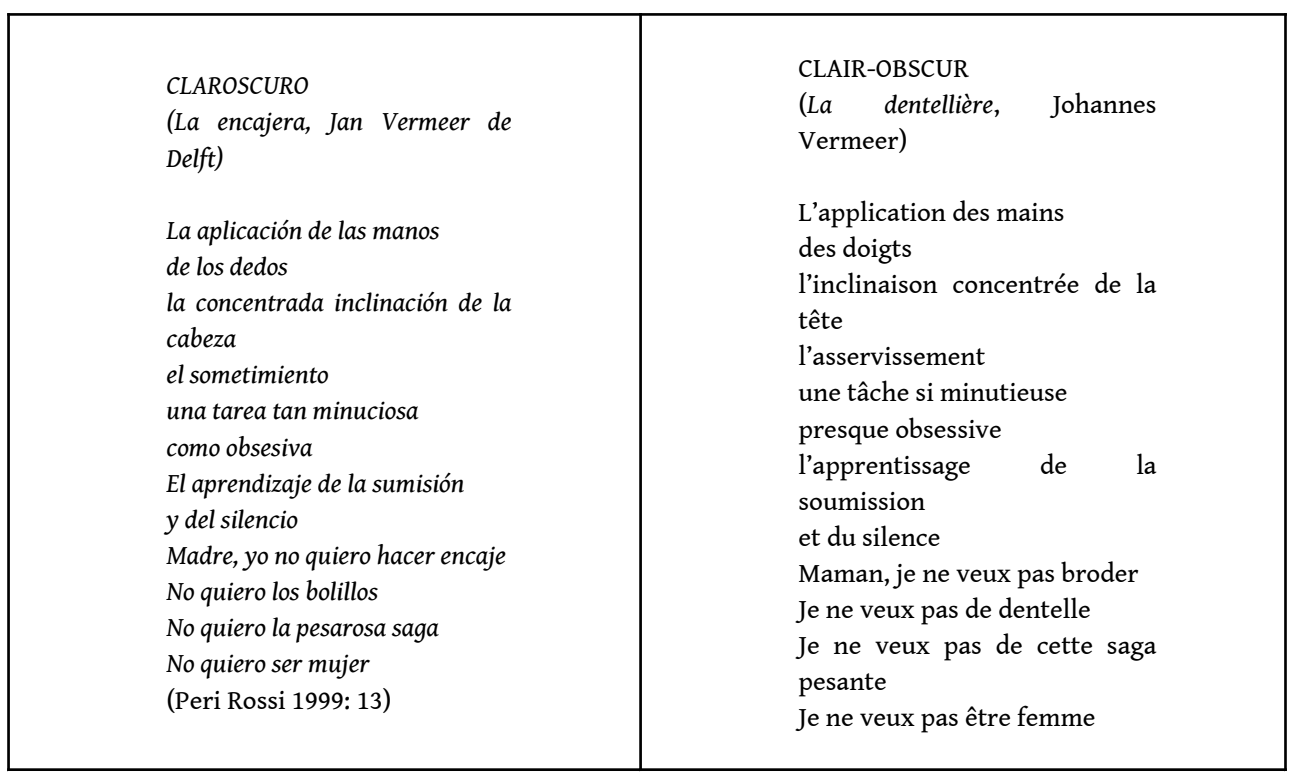

19 A partir d'une réélaboration poétique de «La dentellière ", un tableau du peintre hollandais Jan Vermeer (daté en 1669), Cristina Peri Rossi invente une protagoniste qui expose sans détours ses insubordinations au mandat de genre : pendant qu'elle réalise sa tâche, elle réfléchit et exprime un désir. De façon significative, le mouvement déployé dans le texte conduit de l'extérieur à l'intérieur : en premier lieu, la description externe de la fonction, exercée minutieusement et de manière systématique, nécessitant concentration, et pouvant correspondre à l'exercice de construction de l'identité que les individus intériorisent au travers de l'éducation et de tout l'ensemble de pratiques socioculturelles associées; en deuxième lieu, le sentiment et la voix du sujet qui refuse d'entretenir sa condition. Ses mots la dirigeront vers sa mère ${ }^{10}$, de qui elle hérite la saga, "pesante " dans le double sens de lourde et de génératrice de souffrance.

La mère représente le lien avec la lignée, avec laquelle elle n'a pas coupé les ponts, bien qu'elle l'ait fait souffrir et qu'elle en ait probablement été reniée. La fille le déclare à voix haute, comme dans un poème d'Alfonsina Storni, qui a fait couler beaucoup d'encre, «Bien puediera ser... »" («ça pourrait bien être ...»). Le texte de Peri Rossi dégage une certaine volonté, et la négation est améliorée par l'anaphore insistante des quatre derniers vers, sur lesquels s'appuie le discours direct de la protagoniste, à nouveau dans un mouvement de l'extérieur vers l'intérieur: de la négation d'une fonction - dentellière - à la négation d'une autre - procréatrice, femme - ; du produit de la fonction et de ses instruments (la dentelle, le fuseau) à la filiation (la saga féminine) et finalement au signe. Parce que le mot "femme", sans déterminant, représente le signe, cet ensemble d'attributs que la culture concentre dans une unité représentative, un horizon d'attentes auxquelles elle oblige les sujets à s'ajuster, c'està-dire le stéréotype ; jusqu'au point où, à un degré extrême, le signe puisse substituer le sujet. Dans ...Y a otra cosa mariposa (...Passons à autre chose), l'argentine Susana Torres Molina utilise cette stratégie pour dévoiler des positions hautement codifiées en relation avec le genre, la sexualité et le désir, les trois questions autour desquelles se construit son texte théâtral (Fariña Busto 2005).

21 Dans « La encajera » (« La dentellière »), la mère se tait. On ne lui accorde pas de voix, le portrait de la dentellière occupe tout l'espace, tant dans le tableau que dans le poème. 
Dans celui-ci, l'étatisme de la peinture se rompt en donnant la parole au personnage, décision qui, comme je viens de le commenter, a des effets transcendants. La dentellière ( « encajera ») ne veut pas de ce destin (d'asservissement, de soumission, de silence), ne veut pas s'adapter ("encajarse »). De cette manière, la sémantique hispanophone du mot «encaje» («dentelle») active des signifiés imprévus : nous ne pouvons plus le comprendre seulement comme le tissage élaboré patiemment à l'aide des fuseaux, nous lisons avant tout l'adaptation violente que la voix du poème rejette en bloc: «Je ne veux pas être femme ${ }^{12}$.

Paradoxalement, cet attribut, être une femme, peut se transformer en son opposé. C'est ainsi que le juge le sujet poétique de Género femenino (Genre féminin), recueil de poèmes de la chilienne Teresa Calderón :

\begin{tabular}{|l|l|}
\hline SER MUJER & ÊTRE FEMME \\
Terminé como no sabía & \\
que querría terminar & J'ai terminé comme je ne savais pas \\
Me derroté a mí misma & que j'aurais aimé terminer \\
Y obtuve la única victoria & Je me suis vaincue moi-même \\
A fuerza de costalazos & Et j'ai obtenu la seule victoire \\
me hice hombre & À force de tomber \\
(Calderón, 1989:13) & je suis devenue homme \\
\hline
\end{tabular}

Être et devenir: deux verbes importants au sein des théories féministes et de la sexualité. En effet, que signifie "être femme » ou « être homme » au-delà du mandat inscrit dans une culture construite sur un binarisme coercitif, frustrant et invivable? Au-delà de cela, sûrement rien, puisque les sujets se revendiquent différents, traversés par de multiples variables impossibles à démonter, car elles s'additionnent, s'enchainent et se croisent. Il est évident qu'il existe une vie en dehors des logiques duales, qui sont des simplifications fallacieuses de l'existence. Cependant, les choses sont beaucoup plus compliquées, étant donné que notre vie se développe au sein d'une histoire sociale, culturelle et symbolique, qui a doté le sexe de transcendance et a fait du contrôle de la sexualité féminine l'un de ses principes. Je reprends les mots de Judith Butler: «Les femmes et les hommes existent, pourrait-on dire, en tant que normes sociales, et ils sont, selon la perspective de la différence sexuelle, le contenu de la différence sexuelle» (Butler 2006: 239). Comme les normes sociales exercent une pression, elles sont obligeantes, mais pas compactes; elles ont beau se montrer profondes dans l'espace et dans le temps, il n'y a pas de raison de penser qu'elles manquent de date d'expiration. Elles ne sont pas compactes parce que l'ajustement strict à l'idéal (femme/homme, féminité/masculinité) provoque une impossibilité. Par conséquent Judith Butler considère que les actes du genre se rapprochent de cet idéal. En prenant comme référence la performativité du drag queen, elle affirme : « certains de ces dits hommes pouvaient faire la féminité bien mieux que je ne le pourrais, le ferais ou le voudrais jamais » (Ibidem 243), et cet acte, qu'elle nomme «la cessibilité des attributs ", met en évidence à la fois la complexité et la naturalité du genre.

Les normes génériques sont, en effet, des constructions qui obéissent à des valeurs et à des fins; les effets de sa force, comme je l'ai déjà noté, sont visibles: frustration, inégalité, violence. Butler en arrive à parler de « violations » dès lors que les catégories 
sociales sont « imposées de l'extérieur » et « sont, tout d'abord et nécessairement, non choisies » (243). Et elle ajoute :

Mais cela ne signifie pas que nous ayons perdu la capacité de distinguer entre les violations qui nous habilitent et celles qui nous restreignent. Quand les normes de genre opèrent comme des violations, elles fonctionnent comme une interpellation qu'on ne refuse qu'en acceptant d'en payer les conséquences : perdre son emploi, sa maison, ses perspectives de désir ou de vie (Butler 2006 : 243).

L'apprentissage et l'exercice de la subversion constituent, de ce fait, un impératif pour la liberté, malgré et grâce aux conséquences qu'ils impliquent. Dévoiler les procédés que la culture utilise pour inclure et exclure fait partie de ce processus, de même que la désarticulation de tels procédés, ainsi que la conception de nouvelles formules et paris de vie.

\section{Subversion}

La famille est l'une des institutions que le patriarcat a utilisée avec les meilleurs rendements pour la transmission de rôles et de stéréotypes de genre. De cette fonction dérive l'intérêt qu'il a à l'approfondir dans ses significations et ses dynamiques. Il semble évident qu'il le fasse avant tout envers les femmes et, effectivement, nombreuses ont été les écrivaines qui lui ont donné une place centrale. Je reviens à la figure de la mère qui, très bien instruite par les institutions éducatives et religieuses, a pris sur elle le travail d'assujettissement aux obligations féminines. Ce rôle a fait d'elles les cibles préférées des dissidences de leurs filles, qui finissent par leur exprimer leur non-respect. Dans «Poema por la falta de mi madre », («poème par la faute de ma mère) Martha Kornblith écrit :

\begin{tabular}{|c|c|}
\hline $\begin{array}{l}\text { Madre } \\
\text { he de confesarte que además de } \\
\text { haber enterrado a la muñeca } \\
\text { no he cumplido con tus } \\
\text { aspiraciones } \\
\text { de buena ama de casa, madre de } \\
\text { hogar, } \\
\text { hijos, nietos, etcétera } \\
\text { que me convertí en poeta } \\
\text { que es lo mismo que decir } \\
\text { en poeta suicida } \\
\text { y que por eso } \\
\text { juego y seduzco a la muerte } \\
\text { todas las noches } \\
\text { (Kornblith, } 1997: 30)^{13}\end{array}$ & $\begin{array}{l}\text { Mère } \\
\text { je dois t'avouer qu'en plus } \\
\text { d'avoir enterré ma poupée } \\
\text { je n'ai pas respecté tes } \\
\text { aspirations } \\
\text { de bonne ménagère, femme au } \\
\text { foyer, } \\
\text { enfants, } \\
\text { etcétéra } \\
\text { que je suis devenue poète } \\
\text { ce qui revient à dire } \\
\text { poète suicidaire } \\
\text { et que pour cette raison } \\
\text { je joue et je séduis la mort } \\
\text { toutes les nuits }\end{array}$ \\
\hline
\end{tabular}

Dans «la noche oscura » («la nuit obscure»), Rossella di Paolo, péruvienne comme Kornblith, fait le récit du scandale produit par une jeune en abandonnant sa famille pour vivre avec son amant. À travers un intéressant exercice d'intertextualité avec la " Noche oscura del alma » de San Juan de la Cruz, poème qui s'infiltre à proprement parler dans le texte, l'action individuelle se confronte à la norme sociale. Elle y fait correspondre à l'âme protagoniste du texte mystique un sujet féminin parfaitement incarné, qui descend les escaliers de la maison familiale, pendant que les voix de la mère et des frères l'avertissent concernant le déshonneur : « Mi madre gritando en la 
escalera. Mis hermanos / los pelos arrancados / ¡que no lo sepa nadie!» (Luna 2004 : 325) (" Ma mère criant dans l'escalier. Mes frères / les cheveux arrachés / que personne ne le sache !»). La scène se déroule à Lima, à l'époque actuelle; la solitude silencieuse qui accompagne les mouvements de l'âme dans le poème de San Juan est substituée par le vacarme de la famille et par le monde de la ville :

\begin{tabular}{|l|l|}
\hline En la noche dichosa & $\begin{array}{l}\text { Dans l'heureuse nuit } \\
\text { en secret pour que personne ne me } \\
\text { en secreto que nadie me veía } \\
\text { en un taxi negro hacia otra }\end{array}$ \\
habitación & $\begin{array}{l}\text { dans un taxi noir il y avait une } \\
\text { autre chambre }\end{array}$ \\
sin otra luz que mi rabia por & sans autre lumière que ma rage de \\
vivir & vivre \\
y escribir lo que viviera & et d'écrire ce que je vis ${ }^{14}$ \\
\hline
\end{tabular}

Le désir amoureux, mais surtout celui de liberté, guident la jeune, qui fuit en prenant avec elle quelques objets, dont la machine à écrire qui lui permettra d'immortaliser cette expérience ${ }^{15}$. Cette facette d'écrivaine, productrice d'expériences considérées comme dignes d'être racontées, fait partie de la rébellion et apparait de manière récurrente dans l'œuvre d'un bon nombre d'écrivaines. Non seulement l'expérience est vécue, mais elle est aussi transcrite, de telle manière qu'elle peut se présenter comme un modèle de conduites alternatives.

La manifestation de rupture entre le sujet poétique et ses parents est beaucoup plus osée, virulente et dramatique dans Cuatro esquinas del juego de una muñeca (Quatre angles du jeu d'une poupée, non daté $\left.{ }^{16}\right)$, de la poète guatémaltèque Ana María Rodas. Dans le texte qui ouvre le livre, de format épistolaire, ce sujet, la fille, se retourne contre ceux qui s'étaient montrés disposés à la sacrifier pour obéir fidèlement aux codes patriarcaux. Le geste est d'une subversion ouverte, avec un langage si dévastateur qu'il en arrive à être blessant, comme si c'était la seule manière d'illustrer le comportement également blessant des parents envers leur fille. Faute d'avoir acquis un autre regard, elle deviendrait victime d'un sacrifice, enterrée vivante dans un cimetière de vieux rites et de canons ankylosés.

En raison de l'aspect personnel du ton et la valeur de sa signification, je transcris plus d'un fragment de ce poème en prose : 
Papis queridos: a ustedes quiero aclararles qué es todo esto. Las mujeres me entienden. lo que yo hago no es bueno ni malo. Es mío.

Los veo revolverse, incómodos, en sus poltronas. Presiento que buscan las palabras para invocar los cánones antiguos y tratar de meterme a su yugo nuevamente. ya no es posible. [...]

Papis viejísimos que utilizan la significación de la cultura para forjar cuchillos $y$ clavárselos unos a otros en esa carrera disimulada por llegar primero a la fama $y$ conquistar la eternidad, siempre dentro del juego que ustedes inventaron [...] la antes hija está diciéndoles adiós. [...]

Yo no los necesito. si anduve pegada a los faldones de sus sacos fue porque mi infanciasiendo mujer- se prolongaba artificialmente a través de todas esas cosas que ustedes inventaron para asegurarse que, cuando menos, la mitad de los seres humanos quedaría fuera de la competencia. [...]

Los admiré e hice mías sus ideas por un tiempo y no sabía por qué se me llagaba el cuerpo y el cerebro. ahora entiendo lo infantil de esos propósitos y al ver sus rostros con esta vista nueva que me he dado, comprendo que no pertenezco a este cementerio.

Y me largo"

(Rodas non daté : 9-11)
Mes parents adorés: je veux vous clarifier la situation. Les femmes me comprennent. Ce que je fais n'est ni bien ni mal. C'est à moi.

Je vous vois vous retourner, inconfortables, dans vos fauteuils. J'ai le pressentiment que vous cherchez vos mots pour invoquer vos vieux idéaux et essayer de me mettre à nouveau sous leur joug. Ce n'est plus possible.

Mes vieux parents qui exploitent la signification de la culture afin de forger des couteaux et vous les planter l'un à l'autre dans cette course dissimulée pour arriver en premier à la célébrité et conquérir l'éternité, toujours dans le jeu que vous avez inventé [...] celle qui était autrefois votre fille vous dit adieu. [...]

Je n'ai pas besoin de vous. Si je suis restée collée aux pans de vos vestes, c'est parce que mon enfance -en tant que femme- se prolongeait artificiellement par le biais de tout ce que vous avez inventé pour vous assurer qu'au moins la moitié des êtres humains resterait hors compétence. [...]

Je vous ai admirés et j'ai fait miennes vos idées pendant un certain temps, et je ne savais pas pourquoi mon corps et mon cerveau se couvraient de plaies. Maintenant je comprends à quel point cet objectif était infantile et quand je vois vos visages avec cette nouvelle vision que je me suis créée, je comprends que je n'appartiens pas à ce cimetière.

Et je me tire. («L'expérience la plus atroce de l'enfance est la soumission. / Presque équivalente à l'irréparable»), affirme Luisa Futoransky (2000: 8). Soumission par ignorance, par incapacité à répondre, par confiance en ceux qui se représentent comme des sources d'autorité. Rodas précise: "siendo mujer» (« en tant que femme»), la soumission dépasse le temps de l'ignorance, devient le destin culturellement fabriqué. Un joug. Du rose au blanc: de la couleur des habits du bébé à la couleur de la robe de mariée. Elle marque un rite de passage à une nouvelle forme de domestication. Nous pouvons relire la narratrice de Rosario Castellanos ou écouter l'énonciatrice d'un poème de Vidaluz 
Meneses (Nicaragua 1944). Dans celui-ci, l'usage de références bibliques parait adoucir le message, qui, malgré tout, exige du sujet féminin l'adéquation aux vertus que la pensée chrétienne a construit pour l'entraver. Les termes du texte (travailleuse, prudente et prolifère) ne signifient rien d'autre que le soin du foyer (extensible à tout le milieu aujourd'hui nommé éthique des soins cliniques), soumission et procréation; bien qu'en ayant contracté d'autres, le sujet poétique reconnait qu'il a pu se détacher de ces obligations culturelles qui le suivent et avec lesquelles il maintient une tension permanente ; elles sont toujours ici, à lui rappeler qu'il n'arrive jamais à leur donner entière satisfaction :

\begin{tabular}{|c|c|}
\hline $\begin{array}{l}\text { Cuando yo me casé } \\
\text { la capilla era chiquita } \\
\text { y Monseñor recitó los salmos } \\
\text { de rigor: } \\
\text { "Que sea hacendosa como } \\
\text { Martha, } \\
\text { prudente como Raquel, } \\
\text { de larga vida y prolifera como } \\
\text { Sarah" } \\
\text { y heme aquí tenue sombra de } \\
\text { Martha, } \\
\text { martillando la máquina de } \\
\text { escribir en la oficina } \\
\text { después de los afanes del } \\
\text { hogar, } \\
\text { callando la protesta fútil } \\
\text { "silenciosa Raquel" } \\
\text { transcurriendo mi vida } \\
\text { interminable como un río } \\
\text { para completar a Sarah (1967) } \\
\text { (López Brun 1989: 21) }\end{array}$ & $\begin{array}{l}\text { Quand je me suis mariée } \\
\text { la chapelle était petite } \\
\text { et Monseigneur récitait les } \\
\text { psaumes de rigueur: } \\
\text { "Sois travailleuse comme } \\
\text { Martha, } \\
\text { prudente comme Raquel, } \\
\text { et que ta vie soit longue et } \\
\text { prolifère comme celle de Sarah" } \\
\text { Et me voilà ici, faible ombre de } \\
\text { Martha, } \\
\text { à marteler la machine à écrire } \\
\text { dans le bureau } \\
\text { après les ardeurs du foyer, } \\
\text { à taire la protestation futile } \\
\text { "Raquel silencieuse " } \\
\text { à écouler ma vie interminable } \\
\text { comme une rivière } \\
\text { pour compléter Sarah (1967) }{ }^{17}\end{array}$ \\
\hline
\end{tabular}

Pour le sujet d'Ana María, il n'y a pas de possible périphrase. Comme indiqué plus haut, ses mots sont directs, durs, sans concession d'aucun type ; ils tentent de se constituer en corrélation discursive de ce qui a déjà été décrit. Ce faisant, ils rendent compte de la façon dont la conscience de la soumission (à la domesticité, entre autres, comme levier pour d'autres soumissions) et son dévoilement dans le poème constituent une autre facette de la subversion ${ }^{18}$. Dans Poemas de la izquierda erótica (Poème de la gauche érotique), avec lequel s'est ouvert le chemin de l'histoire littéraire guatémaltèque, elle écrit : 


\begin{tabular}{|l|l|}
\hline $\begin{array}{l}\text { Limpiaste el esperma } \\
\text { y te metiste a la ducha } \\
\text { Diste el manotazo al testimonio } \\
\text { pero no al recuerdo. }\end{array}$ & $\begin{array}{l}\text { Tu as essuyé le sperme } \\
\text { et tu t'es mis sous la douche } \\
\text { Ahora, } \\
\text { yo aquí, frustrada, } \\
\text { sin permiso para estarlo } \\
\text { debo esperar }\end{array}$ \\
$\begin{array}{l}\text { y encender el fuego pais pas le souvenir. } \\
\text { y limpiar los muebles } \\
\text { yllenar de mantequilla el pan } \\
\text { (Rodas 1973: 17) }\end{array}$ & Maintenant, \\
& moi ici, frustrée, \\
& sans permission de l'être \\
& je dois attendre \\
\hline
\end{tabular}

33 Au moment de sa publication, le livre a été réceptionné avec animosité. La critique rejetait non seulement l'amertume du langage poétique, mais aussi les thèmes, la dureté et la forme directe des images et des représentations ${ }^{19}$. L'élément le plus dérangeant dérivait certainement $\mathrm{du}$ ton autobiographique des accusations, qui reflétaient le comportement domestique de celui qui, dans le domaine politique, présentait un discours révolutionnaire. Le jeu du titre reprend cette dénonciation: dans ce qu'il y a d'érotique et dans le privé, l'intime, les hommes de gauche ne s'éloignaient en rien des traditions ni des schémas patriarcaux; les politiques proclamées restaient dehors une fois que le révolutionnaire rentrait à la maison :

\begin{tabular}{|l|l|}
\hline Revolucionario: esta noche & Révolutionnaire : cette nuit \\
no estaré en tu cama. & ne serais pas dans ton lit. \\
Que no te extrañe la & Ne soit pas étonné par la \\
subversión de amor & subversion de l'ancien \\
antiguo dueño & amour-maitre \\
Tú hinchas el cuero & Tu luttes contre les oppressions \\
$y$ te preocupas tanto de & et te préoccupes tellement des \\
problemas sociales & problèmes sociaux \\
No te fijas, farsante, & Tu ne te rends pas compte, \\
que en tu casa & imposteur, \\
calcas tan justamente & que dans ta maison \\
los modales del mejor tirano. & tu calques avectant de justesse \\
(Rodas 1973 :86) & les modèles du meilleur tyran \\
\hline
\end{tabular}

Dans un pays qui, à cette époque, était soumis à de fortes tensions politiques, la voix de Rodas a eu un grand impact. Mais l'intérêt du recueil de poème découle des mêmes raisons pour lesquelles il a été critiqué : son langage, sa thématique, le fait de ne pas détacher le personnel du politique, et, en conséquence, de lire dans le politique toutes ses contradictions, ses inerties et ses renoncements.

Un fil sentimental parcourt le livre, le regard d'un sujet qui ressent pertes, abandons et trahisons; mais à aucun moment il ne le fait sous le signe de la soumission ou de l'acceptation. Ce sujet féminin réclame une relation égalitaire, revendique son droit au plaisir et à le dire, de vive voix et à travers l'écriture. 


\section{Transgression ${ }^{20}$}

Le langage codifie la soumission. D'une part grammaticalement, le sujet féminin est considéré comme le sujet marqué ; d'autre part, dans ses significations, le féminin est subsidiaire, minorisé; ce qui convoque, lorsqu'il y a des doublons, toutes les acceptations dénigrantes. Il n'y a rien d'innocent dans le langage. Il offre et construit une perception et une conception déterminée du monde: ce qui se voit et ce qui s'exclut, d'où on s'exprime, qui ou quel est l'axe de référence, comment se définissent les positions et les attitudes.

Le canon patriarcal offre ici la résistance la plus féroce. Il n'y a aucun doute à ce qu'en tant qu'instrument de communication et de connaissance, la langue ne peut pas être plus dynamique. En effet, si la réalité l'est, le langage doit également l'être pour permettre à la réalité d'être découverte. Néanmoins, cette considération s'annule lorsqu'elle se réfère au domaine de la réalité qui affecte les sujets et leur construction générique $^{21}$. Ce n'est pas étrange, par conséquent, que les écrivaines thématisent cette résistance. Je ne parle pas de réfléchir en général au sujet de la langue ou de se servir de ses structures comme référence de situations de la vie ${ }^{22}$, mais de thématiser précisément la codification culturelle du genre.

Concrètement, c'est à cette codification que la poète guatémaltèque Ana María Ardón se réfère dans le texte suivant. Elle y dénonce avec un langage cru la responsabilité de ceux qui ont contribué à fixer et à perpétuer les stéréotypes féminins (dans l'arc limité qui va de vierge à pute, et vice-versa) à travers les traits définitoires des entrées des dictionnaires :

\begin{tabular}{|l|l|}
\hline Tan sólo somos las mujeres; & \\
santas madres vírgenes & Nous les femmes sommes \\
dulces comprensivas, & seulement; \\
viscerales emocionales & des Saintes mères vierges \\
brujas neuróticas histéricas & douces compréhensives \\
sensibileras ingenuas & viscérales émotionnelles \\
liberales & sorcières névrotiques hystériques \\
o putas. & sensibles ingénues libérales \\
Según el diccionario & ou des putes. \\
de la Real Academia & Selon le dictionnaire \\
de los Machos. & de l'Académie \\
Pero, de humanidad & des Machos. \\
¿Qués saben los castrados? & Mais, de l'humanité \\
(Saavedra, 2004: 157) & Que savent les castrés? \\
\hline
\end{tabular}

Ana María Rodas met aussi l'accent sur ces valeurs associées au féminin. Cet horizon symbolique de déconstruction est nécessaire dans la perspective d'une société dans laquelle les différences ne sont pas synonymes d'inégalités, mais de potentialités et de richesse : 


\begin{tabular}{|l|l|}
\hline $\begin{array}{l}\text { La gramática miente } \\
\text { (como todo invento masculino) }\end{array}$ & $\begin{array}{l}\text { La grammaire ment } \\
\text { (comme toutes les inventions } \\
\text { Femenino no es género, es un } \\
\text { adjetivo }\end{array}$ \\
que significalines) inferior, & Le féminin n'est pas un genre, \\
inconsciente, utilizable, & c'est un adjectif \\
accesible, fácil de manejar, & qui signifie inférieur, \\
desechable. y sobre todo & inconscient, utilisable, \\
violable. Eso primero, antes que & accessible, facile à manier, \\
cualquier & jetable. Et surtout \\
otra significación preconcebida. & violable. Ceci en premier, avant \\
(Rodas non daté : 45) & la moindre \\
& autre signification préconçue. \\
\hline
\end{tabular}

Dans une vision plus positive, Cristina Peri Rossi examine la possibilité de transformer ce langage patriarcal hérité en provoquant ${ }^{23}$, avec une nouvelle utilisation, l'ouverture à des dimensions non contemplées, ainsi que le démantèlement de clichés et de servilités. Tout ceci dans plusieurs directions: d'un côté, en rendant visible la conventionalité de la (supposée) valeur générique des formes masculines et, de l'autre, en incorporant de manière normalisée différentes expressions de la sexualité et du désir. Au premier critère, répondent des textes comme ce poème de Inmovilidad de los barcos (« Immobilités des bateaux ») :

\begin{tabular}{|c|c|}
\hline $\begin{array}{l}\text { GÉNERO } \\
\text { Poderosa ley gramatical } \\
\text { obliga a pluralizar en masculino } \\
\text { allí donde el género femenino } \\
\text { predomina } \\
\text { pero no es el único absoluto } \\
\text { contra este despotismo } \\
\text { sentimiento primario de justicia } \\
\text { justifica si yo, al escribir, } \\
\text { especifico: lector, } \\
\text { lectora. } \\
\text { (Peri Rossi 1997: 12) }\end{array}$ & $\begin{array}{l}\text { GENRE } \\
\text { Loi grammaticale puissante } \\
\text { obligeant à pluraliser au } \\
\text { masculin } \\
\text { là où le genre féminin } \\
\text { prédomine } \\
\text { mais ce n'est pas le seul } \\
\text { absolu } \\
\text { contre ce despotisme } \\
\text { un sentiment primaire de } \\
\text { justice } \\
\text { justifie que moi, en écrivant, } \\
\text { je spécifie : lecteur, } \\
\text { lectrice. }\end{array}$ \\
\hline
\end{tabular}

41 Le deuxième constitue un des axes structurants de toute la production de l'autrice, autant poétique que narrative, se révélant particulièrement intéressants par rapport à certains récits des collections La rebelión de los niños (La rébellion des enfants) (1988) et Desastres intimos (Désastres intimes) (1997). Dans ces pages apparaissent le complexe d'ÆEdipe, l'attirance d'un homme adulte pour les amies de sa fille, la violence sexuelle, le fétichisme, le masochisme, la féminité et la masculinité comme rôles non esentialistes, et le lesbianisme. Peri Rossi revient à plusieurs de ces sujets dans Habitaciones privadas (Habitations privées), sa dernière œuvre narrative (2012). Il s'agit d'une gamme importante de peurs et de tabous de la culture en lien avec la sexualité et le(s) désir(s), ainsi qu'en relation avec les dérèglements des rôles de genre.

42 L'autrice démontre une énorme habileté à l'heure de formaliser toutes ces questions, (Fariña Busto 2001), qui sont fondamentales autant dans la vie des personnes et des sociétés, qu'afin que la culture se soit chargée de vigilarlas y castigarlas (les surveiller et les punir). En parler est le premier pas pour les exorciser; le suivant est celui de la 
normalisation, ce que Peri Rossi réussit, en faisant du cas non légitime du lesbianisme ${ }^{24}$ une possibilité de plus. Et je comprends cette normalisation comme un signal évident de transgression, puisque, strictement, il suppose la violation d'une $1 \mathrm{loi}^{25}$, celle de l'hétérosexualité normative du patriarcat.

La clé de la narration est réaliste ; pour inscrire les personnages et le conflit auquel ils devront se confronter, des contextes quotidiens reconnaissables ont été choisis. Ce qui est intéressant, c'est le trajet que réalisent ces personnages et les personnes qui se trouvent dans leur entourage. D'un côté, ils ont besoin de trouver une issue à cette nouvelle circonstance qui, de par sa nature, produit avant tout une perturbation. D'un autre côté, ils doivent adopter une attitude face à celle-ci, et de cette manière, autant la personne qui vit directement le problème que celles avec lesquelles elle est liée affectivement, par le travail ou d'une autre manière, se verront impliquées. Les récits ont une incidence sur le parcours réflexif qui se met en mouvement pour la compréhension du conflit, au-delà de la question de son éventuelle résolution; bien que le fait de le comprendre suppose bel et bien une première phase de résolution, qui est généralement accompagnée par un affaiblissement des troubles initiaux et par un type de transformation, quel que soit son degré. Dans « Una consulta delicada » (« Une consultation délicate »), par exemple, le dialogue entre un patient qui se sent femme et son psychologue débouche sur toute une série de considérations sur l'identité sexuelle, le désir, le plaisir et les rôles. Cette conversation est tournée sur l'idée du déguisement, très pertinente, autant pour explorer les plis de l'identité que pour disséquer les masques de la culture. Les sentiments du patient et ses questions amènent le docteur à diriger son regard vers sa propre vie, et à finir par appliquer à lui-même le conseil qu'il avait donné à son patient, se déguiser, ce qui témoigne de son changement d'attitude.

Deux phrases de cette nouvelle me sont utiles pour conclure cette partie : «le docteur Minnovis était un homme normal, c'est-à-dire qu'il avait peu d'imagination » et « On souffre plus pour des raisons imaginaires que pour des réelles » (1997: 106 et 108). Si les normes de la culture, dont la force réside dans leur valeur symbolique, sont intériorisées sans la moindre discussion, elles produisent l'immobilisme (la normalité) ; s'il y a des fissures dans cette intériorisation, ce qui est le plus fréquent, il en résulte de la souffrance. Si elles peuvent être examinées et comprises comme les constructions qu'elles sont, et si on accepte le défi en connaissant ses conséquences, il sera possible de les dénaturaliser et de les transgresser.

Au banquet de la liberté et des droits, les femmes n'ont pas été invitées; elles ont dû faire irruption, voler, démanteler et construire. Déconstruire l'héritage avec et contre le langage reçu est un exercice plein d'obstacles et de défis. Même maintenant, alors que le voyage de la conscience et de la rébellion a permis de penser et de vivre d'autres manières, les femmes continuent de se demander (nous continuons de nous demander) pourquoi la culture les a punies d'une charge si lourde. Cependant, la question n'obéit pas à la méconnaissance, mais à l'incompréhension, ce qui est beaucoup plus profond. Dans un dialogue provocateur avec la mythologie chrétienne, Regina José Galindo ${ }^{26}$ s'exclame : 


\begin{tabular}{|l|l|}
\hline A MI MADRE & MA MÈRE \\
NO LA PREÑÓ UN ÁNGEL & N'A PAS ÉTÉ FÉCONDÉE PAR UN \\
YO, & ANGE \\
NUNCA HE MULTIPLICADO & MOI, \\
NI SIQUIERA MIGAS & JE N'AI JAMAIS RIEN MULTIPLIÉ \\
NO DIRIJO UN CLUB & PAS MÊME DES MIETTES \\
CON DOCE ADMIRADOERS & JE NE DIRIGE PAS UN CLUB \\
NI HE OTORGADO & DE DOUZE ADMIRATEURS \\
-JAMÁS- & \\
EL INDULTO A UNA & JE N'AI JAMAIS OCTROYÉ \\
PROSTITUTA. & -JAMAIS- \\
QUE ALGUIEN ME EXPLIQUE & LA GRÂCE À UNE PROSTITUÉE \\
POR QUÉ & \\
ENTONCES & QUE QUELQU'UN M'EXPLIQUE \\
CARGO UNA CRUZ. & POURQUOI \\
¡POR FAVOR & ALORS \\
NO QUIERO RESCUCITAR! [Sic] & JE PORTE UNE CROIX \\
(Ávila 2004: 283) & \\
& S'IL VOUS PLAIT \\
& JE NE VEUX PAS RESCUCITER ! \\
\hline
\end{tabular}

De la désobéissance à la transgression, sous différents formats, intonations et degrés, ni Alfonsa Storni, ni Delmira Agustini, ni Julia de Burgos, ni Juana de Ibarbourou, ni Victora Ocampo n'ont été obéissantes ; l'autre Juana, Juana de Asbaje, n'a pas obéi non plus. Ses héritières persistent dans cette habitude: le destin des frontières est d'être franchies, celui des injonctions, d'être démantelées.

\section{BIBLIOGRAPHIE}

ÁNGEL, Albalucía. 1985. « Una autobiografía a vuelo de pájara » Revista Iberoamericana 51 (132) : 453-456.

ÁVILA, Myron Alberto. 2004. Mujer, cuerpo y palabra. Tres décadas de rre-creación del sujeto de la poeta guatemalteca (1973-2003). Madrid : Torremozas.

BARD, Christine. 2000. Un siglo de antifeminismo. Madrid : Biblioteca Nueva.

BOSCH FIOL, Esperanza \& FERRER PÉREZ, Victoria Aurora. 2003. « Fragilidad y debilidad como elementos fundamentales del estereotipo tradicional femenino » Feminismo/s $2: 139-152$. URL : https://rua.ua.es/dspace/bitstream/10045/2972/1/Feminismos_2_09.pdf BUTLER, Judith. 2012 [2006]. Défaire le genre: Nouvelle édition augmentée. Paris, Éditions Amsterdam.

CALDERÓN, Teresa. 1989. Género femenino. Santiago : Editorial Planeta Chilena.

CASTELLANOS, Rosario. 1975 [1971]. Álbum de familia. Mexico : Editorial Joaquín Mortiz. 
DE CASTRO, Rosalia. 2002. Anthologie poétique, traduite du Galicien par José Carlos Gonzàlez. Bedée : Éditions Folle Avoine.

FARIÑA BUSTO, María Jesús. 2001. « Desastres íntimas en Desastres íntimos. Políticas en identidades sexuales ", in (Trans)formaciones de las sexualidades y el género, BENGOECHEA, Mercedes \& MORALES LADRÓN, Marisol (éd.). Alcalá de Henares : Univeristé d’Alcalá, 121-132.

FARIÑA BUSTO, María Jesús. 2005. « El cuerpo como espacio de significación en dos textos dramáticos: ...Y a otra cosa mariposa, de Susana Torres Molina, y Vlad, de Teresa Marichal Lugo ", in Dramaturgas femeninas en la segunda mitad del siglo XX. Espacio y tiempo, ROMERA CASTILLO, José (éd.). Madrid : Visor, 365-374.

FUTORANSKY, Luisa. 2000. París, desvelos y quebrantos, New York : Pen Press.

GONZALEZ, Patricia Elena \& ORTEGA, Eliana. 1983. La sartén por el mango, Puerto Rico : Ediciones Huracán.

JUÁREZ BLANQUER, Aurora. 1978. « Madre y cantiga de amigo » Estudios Románicos 1 : 130-152.

KORNBLITH, Martha. 1997. Sesión de endodoncia, Caracas, Editorial Eclepsidra.

LÓPEZ BRUN, María Eugenia. 1989. ...A puro golpe de amor. Seis poetas contemporáneas de Nicaragua. Mexico : Casa de Las Imágenes.

LUNA, Lola. 1996. Leyendo como una mujer la imagen de la Mujer. Madrid, Anthropos.

LUNA, Lola. 2004. Trilogía poética de las mujeres en Hispanoamérica : Rebeldes. Mexico : Ediciones La Cuadrilla de la Langosta.

MONTENEGRO, Gustavo \& GIL, Gemma. 2006. «Entrevista a Ana María Rodas », in Prensa Libre (2 avril). http://prensalibre.com/pl/domingo/archivo/revistad/2006/abril06/020406/ dfrente.shtml (consulté le 10/11/12)

MORET, Zulema. 2004. Mujeres mirando al sur. Antología de poetas sudamericanas en USA. Madrid: Torremozas.

MURGUIALDAY, Clara. 1990. Nicaragua, revolución y feminismo (1977-89). Madrid : Editorial Revolución.

ORTEGA, Eliana. 1996. Lo que se hereda no se hurta. Ensayos de crítica feminista. Santiago de Chile : Editorial Cuarto Propio.

OYARZÚN, Kemy. 1990. «Beyond Histeria : "Haute Cuisine” and “Cooking Lesson”. Writing as Production » in GUERRA CUNNINGHAM, Lucía (éd.), Splintering Darkness: Latin American Women Writers in Swarch of Themselves. Pittsburgh : Latin American Literary Review Press, 87-110.

PANTÍN , Yolanda. 2001. « Entrar en lo bárbaro. Una lectura de la poesía venezolana escrita por mujeres » Kalathos. Revista cultural, avril-mai. URL : http://www.kalathos.com/abr2001/letras/ pantin/pantin.htm

PERI ROSSI, Cristina. 1988. Cosmoagonías. Barcelona : Laia.

PERI ROSSI, Cristina. 1994. Otra vez Eros. Barcelona : Lumen

PERI ROSSI, Cristina. 1997. Inmovilidad de los barcos. Vitoria-Gasteiz : Bassarai.

PERI ROSSI, Cristina. 1999. Las musas inquietantes. Barcelona : Lumen.

PERI ROSSI, Cristina. 2007. Habitación de hotel. Barcelona : Plaza y Janés.

PERI ROSSI, Cristina. 2012. Habitaciones privadas. Palencia : Menoscuarto. 
ROBERTO SODRÉ, Paulo. 2004. « Entre a guarda e o viço : a madre nas cantigas de amigo galegoportuguesas » Temas Medievales $12: 97-128$.

RODAS, Ana María. 1973. Poemas de la izquierda erótica. Guatemala : Testimonio del absurdo diario ediciones.

RODAS, Ana María. Non daté. Cuatro esquinas del juego de una muñeca, Guatemala : Litografías modernas.

SAAVEDRA , Aurora Marya. 2004. Trilogía poética de las mujeres en Hispanoamérica. Pícaras. Mexico : Ediciones La Cuadrilla de la Langosta.

STORNI, Alfonsina. 1999 (rééd). Obras. Poesia. Buenos Aires : Losada.

STORNI, Alfonsina. 2002 (rééd). Obras. Prosa. Buenos Aires : Losada.

TOLEDO, Aida. 2009 : «Feminismo y subversión en los setenta en Guatemala. Poemas de la izquierda erótica de Ana María Rodas, historia de un libro » Destiempos 19 : 346-358. URL : http:// www.destiempos.com/n19/toledo.htm

VALCÁRCEL, Amelia. 1994. Sexo y filosofia. Sobre « mujer » $y$ « poder ». Barcelona : Anthropos.

ZAVALA , Iris. 2000. « Autobiografía con olvidos » Quimera 192 : 8-15.

ZEITZ, Eileen. 1983. « Técnica e ideología en un cuento de Rosario Castellanos », Actes du $8^{\text {ème }}$ colloque de la Asociación Internacional de Hispanistas, 22-27 aout, AIH 765-771. URL : http:// cvc.cervantes.es/literatura/aih/pdf/08/aih_08_2_090.pdf

\section{NOTES}

1. NDT : Il s'agit, ici et dans tous les cas où je n'indique pas de référence, de ma traduction.

2. Eliana Ortega est éditrice, avec Patricia Elena González, de l'incontournable La sartén por el mango. Encuentro de escritoras latinoamericanas, publié en 1984.

3. Ortega précise également la signification du féminisme en Amérique Latine : " s'autoproclamer féministe en Amérique Latine, c'est s'auto-proclamer différente, hybride, métisse » (1996 : 32-33). Iris M. Zavala se définit ainsi : « Je suis (par construction, et non par essence) une femme, d'un pays colonisé, marxiste (je ne l'ai jamais considéré comme une hérésie, car toute hérésie se termine en religion) et féministe, mais pas orthodoxe, ni fondamentaliste, et je ne milite pas pour un dogme... le féminisme est pluriel et pour moi, il dévoile le chiffrage du symptôme social » $(2000: 14)$.

4. Je me réfère à Un siglo de antifeminismo (Un siècle d'antiféminisme), une collection d'essais dont Christine Bard est l'éditrice. Les différents travaux du livre confrontent les lecteurs aux pires déviances des orthodoxies : les préjugés et l'ignorance, ingrédients qui, ensemble ou séparés, n'ont produit que de l'intolérance, de l'immobilisme, des inégalités et de la violence.

5. La nicaraguayenne Gioconda Belli, alors qu'elle était engagée dans l'activisme révolutionnaire, l'expliquait ainsi : «Je crois que nous avons manqué d'homogénéité et de stratégie dans la mise en place du Front sur le thème de la femme. De plus, l'opinion de certaines femmes influentes a brouillé la compréhension de notre problématique. Il y a eu des erreurs d'interprétation, notamment concernant les perspectives du féminisme : on a vendu l'idée que le féminisme était synonyme de haine envers les hommes, le lesbianisme et la liberté sexuelle, et on a perdu de vue le contenu révolutionnaire du féminisme. C'est problématique, car de nombreux'ses et précieux·ses membres se sont laissée's convaincre par cette publicité mensongère " (Murguialday 1990 : 222). 
6. Il existe beaucoup d'analyses littéraires de cette nouvelle de Rosario Castellanos. Je recommande, par exemple, Oyazún (1990) et Zeitz (1983).

7. En 1841, Gertrudis Gómez de Avellaneda avait déjà établi cette équation (mariage = esclavage) dans son roman Sab.

8. « GENEALOGÍA (Sappho, Virginia Woolf et autres): dulces antepasadas mías / ahogadas en el mar / o suicidas en jardines imaginarios / encerradas en castillos de muros lilas / y arrogantes / espléndidas en su desafío / a la biología elemental / que hace de una mujer una paridora / antes de ser en realidad una mujer / soberbias en su soledad / y en el pequeño escándalo de sus vidas // Tienen lugar en el herbolario / junto a ejemplares raros / de diversa nervadura " (Peri Rossi, 1994: 9) - (GÉNÉALOGIE : mes douces ancêtres / noyées dans la mer / ou suicidées dans des jardins imaginaires / enfermées dans des châteaux aux murs lilas / et arrogants / splendides dans votre défi / envers la biologie élémentaire / qui fait d'une femme une accoucheuse / avant d'être en réalité une femme / splendeur dans sa solitude / et dans le petit scandale de leurs vies // Elles ont lieu dans l'herboriste / avec quelques exemplaires étranges / de différentes nervures).

9. La figure et l'œuvre de Gómez de Avellaneda servent à Marti de contrepoint à celles de Luisa Pérez, à qui est dédiée la chronique où la comparaison entre les deux : « Luisa Pérez de Zambrana ", datée en 1875 et publiée après la mort de l'écrivain.

10. Le rôle de la mère comme destinataire de la voix poétique a une large tradition dans la culture littéraire. Dans le milieu hispanophone, mentionnons notamment les cantigas de amigo et les chansons médiévales, où il est très fréquent qu'une jeune fasse confidence de ses préoccupations et de ses décisions à sa mère, qui parfois la réprimande, mais d'autre fois, l'aide et la conseille. Il est clair que dans ces cas, il s'agit toujours de peines de cœur. A propos, on peut voir Juárez Blanquer (1978) et Roberto Sodré (2004).

11. «A veces a mi madre apuntaron antojos / De liberarse, pero se le subió a los ojos / Una honda amargura, y en la sombra lloró. // Y todo eso mordiente, vencido, mutilado / Todo eso que se hallaba en su alma encerrado, / Pienso que sin quererlo lo he libertado yo. » (« Parfois venait l'envie à ma mère / De se libérer, mais lui montaient aux yeux / Une profonde amertume, et dans l'ombre elle pleurait. // Et tout cela gravé, vaincu, mutilé / Tout cela qui se trouvait enfermé dans son âme / Je pense que sans le vouloir je l'ai libéré moi-même »)

12. Tous les éléments du poème sont parfaitement ajustés, en commençant par cette conclusion où l'absence de déterminant est elle-même déterminante : elle supère toute spécification car elle fait allusion au signe (pas une femme, mais femme).

13. Martha Kornblith est née à Lima en 1959 et décédée à Caracas en 1997. Elle a écrit trois recueils de poèmes : Oraciones para un dios ausente (1995), El perdedor se lo lleva todo (1997), et Sesión de endodoncia (1997). Le poème cité est tiré du troisième.

14. Mise en forme voulue par R. di Paolo. (note de la traductrice)

15. Je me suis référée à ce texte de Rosella di Paolo et a certains textes d'Ana María Rodas, dont je vais parler par la suite, dans un article actuellement en presse : «Hablando de sexo y de placer. Palabra de poetas latinoamericanas » (« En parlant de sexe et de plaisir. Parole de poètes latinoaméricaines »)

16. Le livre a été publié en 1975 , bien que la date ne figure pas dans cette édition.

17. Dans d'autres poèmes (" Recuento »), elle insistera : " Le retour aux habitations crépusculaires / où sublimait, femme du vingtième siècle, / la soumission. / Martha, la travailleuse / Raquel, la prudente / Sarah, celle au ventre florissant au crépuscule » (Ibidem : 36). 18. Peut-être faudrait-il appliquer à l'écriture d'Ana María Rodas le concept de « entrada en lo bárbaro " (« entrée dans le barbare ») qu'utilise Yolanda Pantín pour décrire le langage, la syntaxe et la formulation poétique de certaines poètes vénézuéliennes.

19. L'autrice rappelle : «tout le monde disait que ceci n'était pas de la poésie » (Montenegro: 2006). Mais cette idée était déjà reprise dans un des poèmes, qui y répondait : « Los poetas tienen 
fama / de utilizar palabras suaves. / De hablar del amor, de la melancolía, / de los cielos azules, del horizonte vago. / O yo no soy poeta / o pongo en entredicho a mis colegas / ¡Qué vergüenza que no me dé vergüenza lo que digo! » («Les poètes ont la réputation / d'utiliser des mots doux. / De parler de l'amour, de la mélancolie, / des ciels bleus, de l'horizon vague. / Soit je ne suis pas poète / soit je mets mes collègues en doute. / Quelle honte que je n'ai pas honte de ce que je dis ! ») (Rodas 1973 : 78). Des années après, en 2000, Rodas a reçu le Prix National de Littérature Guatémaltèque. En ce qui concerne la réception de ce livre, Toledo est une bonne référence (2009).

20. Dans cette partie, j'aborde deux questions, le langage et la sexualité, qui d'une certaine façon, soutiennent les apartés précédents. Néanmoins, je me réfère ici au langage en soi comme thème et à des sexualités non normatives. Cela m'intéresse d'offrir cette dimension particularisée comme mode spécifique de transgression.

21. La version en ligne du dictionnaire de la Real Academia Española affiche cette déclaration : « Les langues changent en continu, et elles le font de manière spéciale dans leur composant lexicaux. C'est pourquoi les dictionnaires ne sont jamais terminés : ils sont une œuvre vivante qui s'efforce de refléter l'évolution en enregistrant de nouvelles formes et en assistant aux changements de signification ». Contradictoirement, elle refuse d'enregistrer l'acception du terme « généro » ("genre ») tel qu'il est utilisé au sein des théories féministes. Alors que depuis 2005, le Diccionario panhispánico de dudas (Dictionnaire hispanophone des doutes) la recueille.

22. Cette question exigerait un article entier, car sa présence est récurrente dans un grand nombre de textes. Un exemple exceptionnel est constitué par l'ensemble de l'œuvre de Cristina Peri Rossi, dans tous les genres qu'elle a cultivés. Dans le récit " La syntaxe ", du recueil Cosmoagonías (Cosmo-agonies), elle établit une équivalence entre la dynamique de relation d'une famille et les structures syntactiques : "Douloureusement je me suis rendue compte que les relations les plus profondes se structurent très solidement sous forme de formules rigides et répétitives : l'impossibilité de rompre le lien se manifeste par l'impossibilité de modifier la syntaxe » (Peri Rossi, 1988 : 120-121). Dans la même collection, le récit « La índole del lenguaje » («La nature du langage ») se termine par l'observation suivante : « Le langage appartient à ceux qui ont le pouvoir $»(92)$.

23. « Je parle la langue des conquistadors / mais je dis l'opposé de ce qu'ils disent » (Peri Rossi, $1994: 11)$.

24. En plus de certains récits - «La semaine la plus merveilleuse de nos vies » et « Le témoin », dans Desastre íntimos (Désastres intimes), ou « Se busca » ( Avis de recherche »), dans Habitaciones privadas (chambres privées) -, Cristina Peri Rossi traite, dans tous ses recueils de poèmes, du désir des femmes et du désir entre femmes, que le patriarcat a nié et refuse de comprendre.

25. Selon le Dictionnaire de la Real Academia Española : transgredir 1. Enfreindre, violer un précepte, une loi ou une charte.

26. Regina José Galinda est guatémaltèque, comme Ana María Ardón et Ana María Rodas. Elle a commencé sa trajectoire artistique comme poète, mais se réalise fondamentalement dans le territoire de la performance depuis de nombreuses années.

\section{RÉSUMÉS}

La désobéissance féminine quant aux injonctions culturelles remonte à plus de quelques décennies, bien que sa conceptualisation soit récente. Cet article analyse les contributions 
d'écrivaines hispano-américaines sous différents genres littéraires, dont notamment la poésie. L'objectif est de faire connaitre des œuvres aux qualités réflexives et combatives, des modèles de résistance et de rébellion. Bien que le corpus se réfère principalement aux dernières décennies du vingtième siècle, il inclut également des textes publiés antérieurement.

Women's disobedience towards cultural mandates is not a recent plan of action, nor is it from a few decades ago, even though conceptualization of this kind of disobedience can be considered recent. This article discusses the approaches taken by Latin-American women writers which are materialized in different literary genres, with a certain preference for poetry. The main objective of this paper is to present reflective and challenging wordings as well as models of resistance and rebellion. The textual corpus, which is in chronological order, corresponds to the last decades of the century not excluding, however, some references to earlier relevant texts.

\section{INDEX}

Mots-clés : genre, féminisme, autrices hispano-américaines, désobéissance, transgression Keywords : gender, feminism, Latin-American women writers, disobedience, transgression Thèmes : Recherches

\section{AUTEURS}

\section{MARÍA JESÚS FARIÑA BUSTO}

Université de Vigo

María Jesús Fariña Busto est Professeure de littérature espagnole et latino-américaine à l'Université de Vigo, en Espagne. Docteure en philologie, ses recherches s'inscrivent principalement dans la théorie féministe quant à la représentation du corps, la sexualité et la violence infligée aux femmes. 